\title{
A contact-less 2-dimensional laser sensor for 3-dimensional wire position and tension measurements
}

\author{
Matthias Prall, V. Hannen, R. Jöhren, H.W. Ortjohann, M. Reinhardt, \\ and $\mathrm{Ch}$. Weinheimer \\ All authors are from \\ Institut für Kernphysik, University of Münster, Germany \\ Email: matthias.prall@uni-muenster.de \\ M. Reinhardt's present address is: \\ Institut für Biologische Grenzflächen \\ Forschungszentrum Karlsruhe, Germany \\ To be published in the april 2010 issue of \\ IEEE Transactions on Nuclear Science \\ DOI identifier 10.1109/TNS.2010.2042612
}

July 25, 2018 


\begin{abstract}
We have developed a contact-less two-dimensional laser sensor which combines position and tension measurements of wires with a diameter of order $0.2 \mathrm{~mm}$. The sensor consists of commercially available laser pointers, lenses, color filters and photodiodes. In our application we have used this laser sensor in conjunction with an automated three-dimensional coordinate measuring machine (CMM). The device allows for a position measurement of wires in three dimensions with an accuracy of about $10 \mu \mathrm{m}$. At the same time the wire tension can be determined with an accuracy of $0.04 \mathrm{~N}$. The device is operated at a distance of $150 \mathrm{~mm}$ from the wire.

For each position measurement, the laser sensor is moved by the automated CMM into a plane, where the coordinates at which the wires intersect with this plane are determined. The position of the plane itself (the third coordinate) is given by the third axis of the CMM which is perpendicular to this plane. The precision of the device was determined using stainless steel wires with a diameter of $0.2 \mathrm{~mm}$ and a tension of $5 \mathrm{~N}$. We use the sensor for quality assurance of the wire electrode modules for the KATRIN neutrino mass experiment. These modules are comprised of two layers of wires, which are $70 \mathrm{~mm}$ apart. In general, the device presented here is well suited for the measurement of any complex wire chamber geometry.
\end{abstract}

\title{
I Introduction
}

The main spectrometer of the KATRIN neutrino mass experiment [1] will be equipped with 248 wire electrodes (Fig. (1). These wire electrodes consist of two layers of stainless steel wires, which are approximately $70 \mathrm{~mm}$ apart. The diameters of the wires are $0.2 \mathrm{~mm}$ and $0.3 \mathrm{~mm}$, respectively for the two layers. We have developed a special laser sensor, which provides the measurement of the wire positions and their respective tensions in both layers. The instrument had to meet the following specifications: 1) Measurement from a distance of $150 \mathrm{~mm}$ to avoid the necessity to move through a wire layer during a measurement, 2) position measurement with an accuracy of at least $0.1 \mathrm{~mm}$ for wires with a diameter of $0.2 \mathrm{~mm}$ and $0.3 \mathrm{~mm}, 3$ ) tension measurement with an accuracy of better than $0.1 \mathrm{~N}$ to assure that wire tension is less than $1 \mathrm{~N}$ above a critical value, 4) total weight of less than $250 \mathrm{~g}$, and, 5) operation under clean room conditions.

With the ascent of wire chambers in nuclear and particle physics many methods have been developed to precisely measure the position and tension of thin wires ([3], [4], [5], [6], [7], [8]). Based on this experience our developments focused on the above requirements.

The basic idea of our method is the use of two laser beams of different wavelength which allows for a very fast kind of triangulation of the position of the wire in two dimensions. The lasers and the corresponding light detectors are precisely moved with a three-dimensional coordinate measurement machine (CMM) to allow an overall threedimensional position determination. When the wire is excited to oscillate by a puff of clean gas, the Fourier transform of the reflected light signal is used to determine the wire tension. The combination of these methods is new and fulfills all the mentioned 


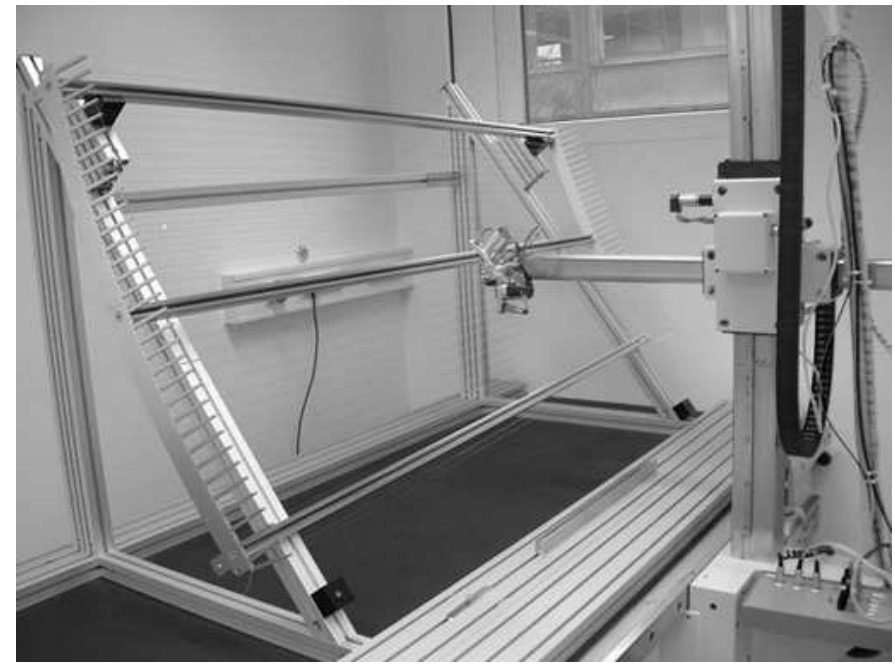

Figure 1: Three-dimensional coordinate measuring machine with the laser sensor and a KATRIN electrode module with two wire layers in our class 10000 clean room [2] at the University of Münster. Here, the sensor is being moved upwards during a positionmeasurement.

requirements. Our device could be well suited for the measurement of a complex detector which consists of many wires e.g. a large wire chamber.

Our article is organized in the following way: in Section II, we describe the setup of our laser sensor. Section III covers the principle and accuracy of the position measurement with our device. In Section IV the tension measurement is described. In Section $\mathrm{V}$ we discuss sources of systematic errors. Finally, in Section VI and VII we give a comparison to other methodes and our conclusions. 


\section{Set-Up of the Laser Sensor}

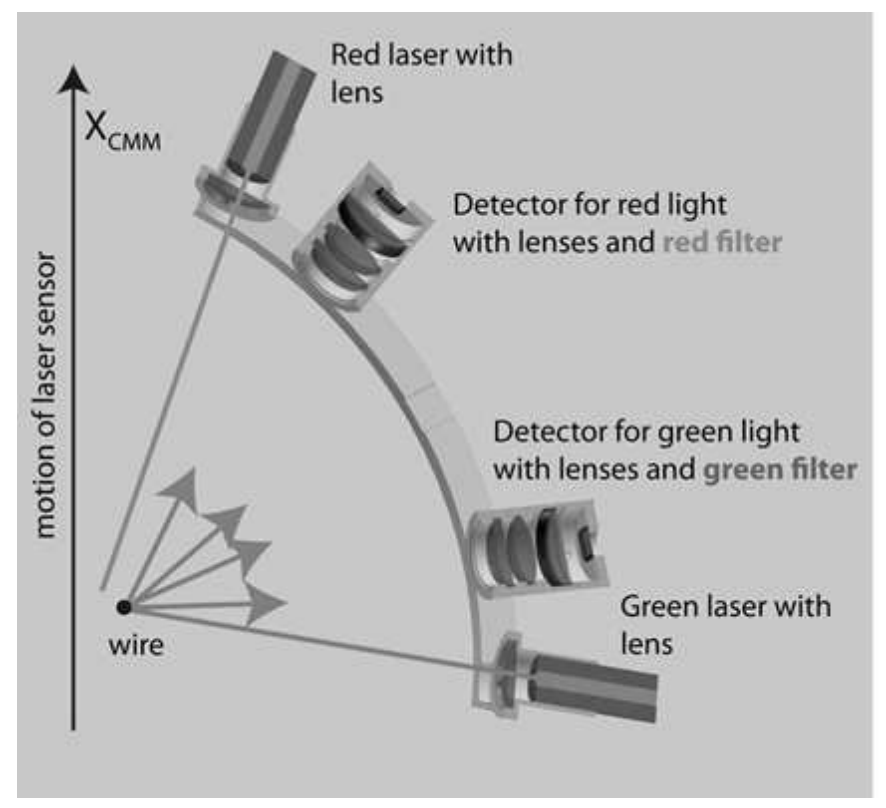

Figure 2: Cross-sectional view of the laser sensor. Filters are mounted directly in front of the diodes to ensure that the PIN diodes detect only red or green light.

Figure 2 shows our contact-less two-dimensional laser sensor. The laser beams, which are generated by ordinary laser pointers with $1 \mathrm{~mW}$ power have diameters of approx. $2 \mathrm{~mm}$ at the exit of the laser pointers. The wavelength of the green laser is $532 \mathrm{~nm}$. The wavelength of the red laser pointer is in the range of $640 \mathrm{~nm}$ to $660 \mathrm{~nm}$. The battery compartments of the laser pointers were removed (Fig. 3) and the batteries were replaced by cables connected to a laboratory power supply (Fig. 3). The removal of the batteries also led to a reduction in deformation of the laser mount due to the weight of the batteries. In order to make precise position measurements with our detector, the laser beams need to have diameters which are small compared to the diameter of the wire. To meet this requirement we focus the laser beams with lenses ( $\mathrm{f}=150 \mathrm{~mm}$, diam. $22 \mathrm{~mm}$ ), where the focal length corresponds to the desired distance between the laser and the wire. The intersection point of both laser beams coincides with the focal points of both lasers.

The light reflected from the wire is detected with PIN diodes. The special optics of the light detectors (Fig. (4) has the purpose to focus as much light as possible onto the PIN diode. The first lens $(\mathrm{f}=150 \mathrm{~mm}$ ) parallelizes the light, which is reflected from the wire. The second lens $(f=20 \mathrm{~mm})$ focuses the light onto the PIN diode. Green and red bandpass filters [10] sit in front of the PIN diodes. The light detectors need to be equipped with filters such that they detect only red or green light. The filters ensure that the device can be operated under standard light conditions and that crosstalk between 


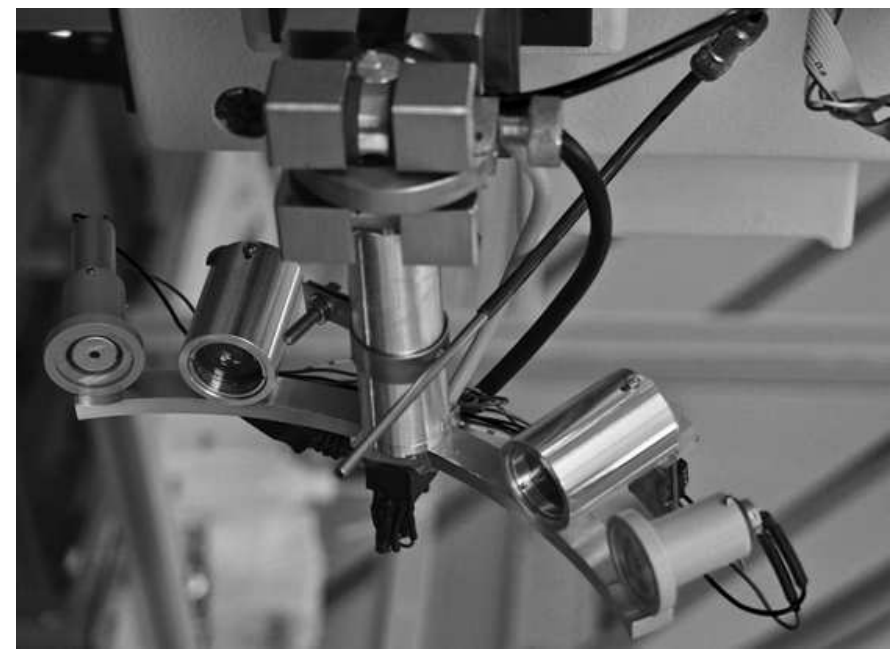

Figure 3: The laser sensor with its most important components. A: the red laser, B: the detector for red light, C: the gas nozzle for the excitation of the wire, D: the detector for green light and $\mathrm{E}$ : the green laser.

the two different reflection signals can be excluded. The signal of the PIN diodes is amplified by a custom-made operational amplifier circuit and recorded by a digitizer card from National Instruments [11].

For the position measurement the laser sensor is moved by a three-dimensional coordinate measurement machine $(\mathrm{CMM})$ across the wire at a distance of $\approx 150 \mathrm{~mm}$ and to detect the laser light, which is is reflected from a wire (Fig. 2). This distance needs to be within $5 \mathrm{~mm}$ of the focal points due the use of the focusing optics. Figures 1 and 3 show the sensor mounted on the measurement head of our CMM. Our CMM is a 20 years old and very robust machine (weight $2500 \mathrm{~kg}$, System C by Stiefelmayer, Fig. 11). It has been equipped with three motors (type A-Max by Maxon Motor [9]) for the three axes and modified to make it compliant with the operation in a class 10000 clean room [2].

Due to the use of a very robust CMM and speed-regulated direct current motors we could avoid vibrations of the laser sensor which would otherwise affect the precision of the measurements. The motors are controlled with LabVIEW via three RS-232 interfaces of a PC, which also digitizes the signals from the laser sensors. The complete software was written with LabVIEW, version 8.0 [12]. The three-dimensional position of the measurement arm, which holds the laser sensors is measured with three magnetomechanical encoders with a resolution of $0.01 \mathrm{~mm}$. The position information of these three encoders is recorded with LabVIEW via the measurement card. The requirement for the laser sensor to be light-weight results from the maximum load specification of $250 \mathrm{~g}$ for a sensor on the CMM, which does not compromise the specified precision. 


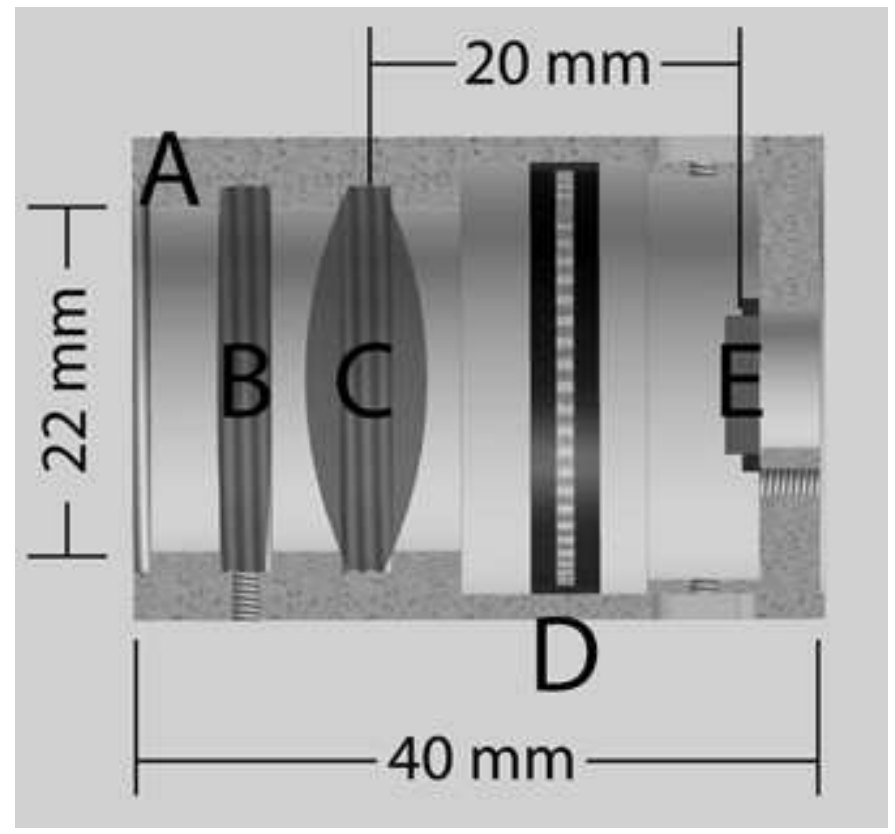

Figure 4: Cross-sectional view of a light detector for a reflection. A: housing, B: lens $f=150 \mathrm{~mm}, \mathrm{C}$ : lens $\mathrm{f}=20 \mathrm{~mm}, \mathrm{D}$ : red or green filter, E: PIN-diode

\section{Position Measurement}

In this section we explain the principle of position measurement of a wire with our laser sensor. The coordinates $x$ and $y$ of the wire will be determined by the sensor (Fig. 5) by moving it across the wire in $\mathrm{x}$-respectively $\mathrm{y}$-direction and recording the $\mathrm{x}(\mathrm{y})$-positions $x(y)_{\text {green }}$ and $x(y)_{\text {red }}$, when the reflections of the green and red lasers from the wire hit the detectors. The z-coordinate is defined by the CMM-position of the laser sensor.

The basic $(x, y, z)$ coordinate system is defined by the CMM encoders. For a given $z$, a fixed point $\left(x_{0}, y_{0}\right)$ can be set by setting the sensor such, that a reference wire on the device to be scanned is at the intersection of the two laser beams. The angles of the two laser beams have to be calibrated beforehand. For this, the sensor has to be moved across a wire at different y-distances (e.g. $5 \mathrm{~mm}, 10 \mathrm{~mm}, 15 \mathrm{~mm}, 20 \mathrm{~mm}$ ). The angles of the laser beams can then be calculated by fitting the distance versus the position of the maximum of the reflection in CMM coordinates $x_{\text {green }}\left(x_{\text {red }}\right)$.

The position $\left(x_{\text {wire }}, y_{\text {wire }}\right)$ of any other wire can be calculated using this calibration point and the inclinations of the laser beams $a_{\text {green }}$ and $a_{\text {red }}(\delta x / \delta y=a)$, where $a_{\text {green }}=-1 / \tan \beta$ and $a_{\text {red }}=1 / \tan \alpha$ (cf. Fig. [5), as follows:

$$
\begin{aligned}
& y_{\text {wire }}=y_{0}-\frac{x_{\text {red }}-x_{\text {green }}}{a_{\text {red }}-a_{\text {green }}} \\
& x_{\text {wire }}=x_{0}-\frac{a_{\text {red }} \cdot x_{\text {green }}}{a_{\text {red }}-a_{\text {green }}}
\end{aligned}
$$




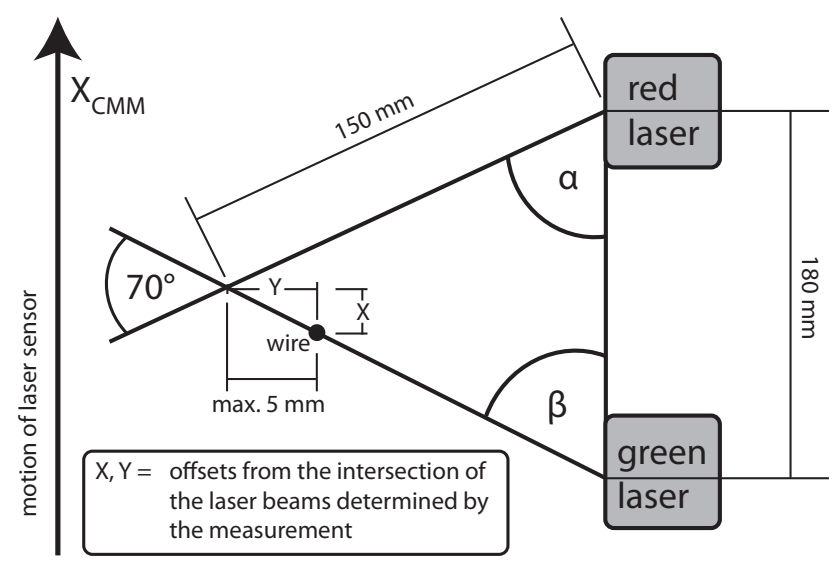

Figure 5: Determination of the y-coordinate of the wire. In our case the angle between the two laser beams amounts to $70^{\circ}=180^{\circ}-\alpha-\beta$. This can be changed for other applications.

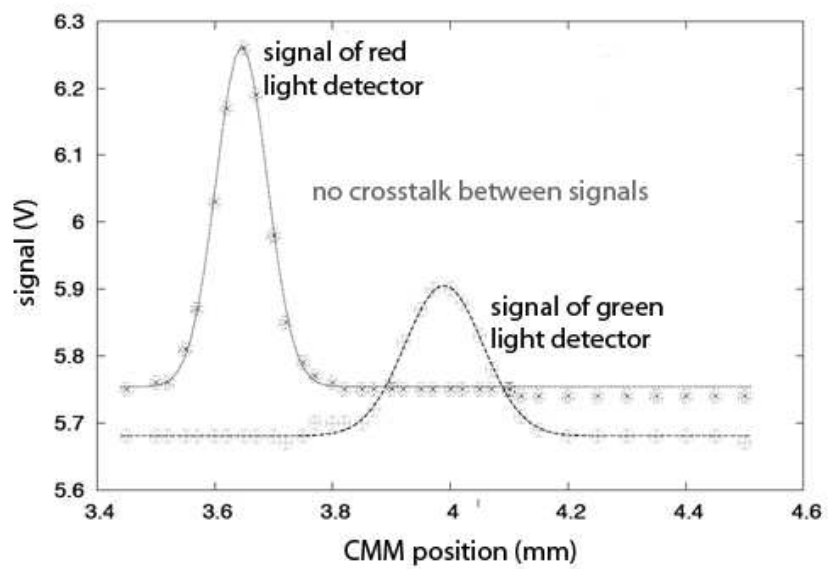

Figure 6: Reflections of the red and green laser as measured with the laser sensor. The position information is derived from the CMM, which moves the sensor across the wire. 
In order to determine the accuracy of the position measurement with the laser sensor, we have mounted a test wire with a diameter of $0.2 \mathrm{~mm}$ in a set-up which could be moved in both $\mathrm{x}$ - and $\mathrm{y}$-direction by means of micrometer screws. Table 1 shows the results of these tests. Positions with index wire were set using the micrometer screws. Positions with index meas were determined with the laser sensor. The resolution of the CMM which moves the sensor is $0.01 \mathrm{~mm}$. To estimate the precision of the laser sensor, we assume that the relation between the wire positions and the measured positions can be described by the equations:

$$
x_{\text {meas }}=b_{x}+s_{x} \cdot x_{\text {wire }} \quad y_{\text {meas }}=b_{y}+s_{y} \cdot y_{\text {wire }} .
$$

Table 1: Settings used for the determination of the accuracy of the position measurements with our laser sensor. Two independent series of measurement were taken for $\mathrm{x}$ and y, respectively. Only one coordinate was varied for each set of measurements.

\begin{tabular}{cc|cc}
$x_{\text {wire }}(\mathrm{mm})$ & $x_{\text {meas }}(\mathrm{mm})$ & $y_{\text {wire }}(\mathrm{mm})$ & $y_{\text {meas }}(\mathrm{mm})$ \\
\hline \hline 0.00 & 0.09 & 0.30 & 0.34 \\
0.10 & 0.20 & 0.40 & 0.42 \\
0.20 & 0.30 & 0.50 & 0.54 \\
0.30 & 0.39 & 0.60 & 0.62 \\
0.40 & 0.50 & 0.70 & 0.72 \\
0.50 & 0.60 & 0.80 & 0.82 \\
0.60 & 0.69 & 0.90 & 0.92 \\
0.70 & 0.79 & 1.00 & 1.02 \\
0.80 & 0.89 & 1.10 & 1.11 \\
0.90 & 0.99 & & \\
1.00 & 1.09 & & \\
\hline
\end{tabular}

There can be arbitrary offsets $b_{x}$ and $b_{y}$ between the laser sensor data and the actual positions. The offsets result from the initial position of the micrometer screw which moved the test wire. The slopes $s_{x}$ and $s_{y}$ indicate the size of the systematic error.

Equations (2) were fitted to the data (Table 1 ) by a least-squares fit under the assumption of a constant uncertainty $\sigma_{x}\left(\sigma_{y}\right)$ for the x- and y-determination by the laser sensor. This resulted in uncertainties of $\sigma_{x}=0.005 \mathrm{~mm}$ and $\sigma_{y}=0.007 \mathrm{~mm}$, respectively.

The uncertainty of the measurement of both coordinates is smaller than the smallest encoder step $s_{\min }=0.01 \mathrm{~mm}$ of the CMM. This is reasonable as the statistical limit $L$ for the statistically distributed difference $\left|x_{\text {wire }}-x_{\text {meas }}\right|$ is

$$
L=s_{\min } \frac{1}{\sqrt{12}}=0.003 \mathrm{~mm} .
$$

The results in Table 2 show the precision of the position measurements at a distance of less than $1 \mathrm{~mm}$ from the intersection of the two laser beams. Especially the slope of $s_{y}=0.973 \pm 0.009 \mathrm{~mm} / \mathrm{mm}$ could be due to scale errors of the micrometer 
Table 2: Best fit values for the test of the precision of the position measurement

\begin{tabular}{lcc} 
& Fit of X values & Fit of Y values \\
\hline \hline offset $b(\mathrm{~mm})$ & $0.097 \pm 0.003$ & $0.042 \pm 0.007$ \\
slope $s(\mathrm{~mm} / \mathrm{mm})$ & $0.993 \pm 0.005$ & $0.973 \pm 0.009$ \\
\hline
\end{tabular}

screw. However, it is also possible that the deviation of $s_{y}$ from unity points towards a systematic uncertainty in the determination of the position of the wire far away from the intersection of the laser beams. This shows that the distance between the wire and the intersection point has to be minimized in order to guarantee a precision of about $10 \mu \mathrm{m}$.

\section{Tension Measurement}

When a wire of length $l$, density $\rho$ and cross-sectional area $A$ is stretched by a force $F$ between two fixed ends, is excited in transverse direction, it will oscillate. The fundamental mode has a wavelength of $\lambda=2 \cdot l$. The speed of sound of a wave on such a wire is given by

$$
c=\sqrt{\frac{F}{A \rho}} .
$$

With $c=\lambda f$, the oscillation frequency $f$ of the fundamental mode of the wire is given by:

$$
f=\frac{1}{2 \cdot l} \sqrt{\frac{F}{A \cdot \rho}}
$$

For small oscillation amplitudes this formula is correct to good approximation, since corrections for the elasticity of the material can be neglected.

We use Eq. (5) to determine the tension of the wires in our electrode modules with our laser sensor. The design value is $10 \mathrm{~N}$ for wires with $0.3 \mathrm{~mm}$ diameter and $1.8 \mathrm{~m}$ length. The material of the wires is stainless steel type $1.4404\left(\rho=7980 \mathrm{~kg} / \mathrm{m}^{3}\right)$. Thus the oscillation frequency is expected to be around $37 \mathrm{~Hz}$.

The frequency is determined through measurement of the time-dependent reflection amplitude of one of the laser beams, e.g. the red one. Note that the wire will reflect light twice per oscillation period, thus the reflected light pattern has a frequency $f^{\prime}=2 \cdot f$. We always observed the lowest frequency at this frequency. Higher harmonics always appear at multiples of $f^{\prime}$. We observed that these always exist in our measurements. The first harmonic is often dominant directly after the excitation and dies out quickly. The wire has to be close to the intersection of the two laser beams, because the diameter of the laser beam needs to be small. The wire starts to oscillate after it was excited by a short puff of clean gas (Ar 4.6). A single pulse is sufficient since even a small 
displacement of the wire results in a clearly detectable signal. The nozzle, which is used to direct that puff at the wire is labeled with ' $C$ ' in Fig. 3. The nozzle consists of a metal capillary with an inner diameter of $2 \mathrm{~mm}$. An electronically controlled valve opens the connection between the nozzle and a reservoir of argon with a pressure of 8 bar for a duration of $10 \mathrm{~ms}$. This results in a puff which has a diameter of approx. $10 \mathrm{~mm}$ at the location of the wire. The distance which the argon has to travel between the valve and the exit of the nozzle is approximately $130 \mathrm{~mm}$ (cf. Fig. 11 and Fig. 3). One can also excite wire oscillations with a mechanical device, e.g. a small hammer, without damaging the wires. However, our method is entirely contact-less. The versatility of the CMM avoids the necessity to adapt any mechanics for different module geometries.
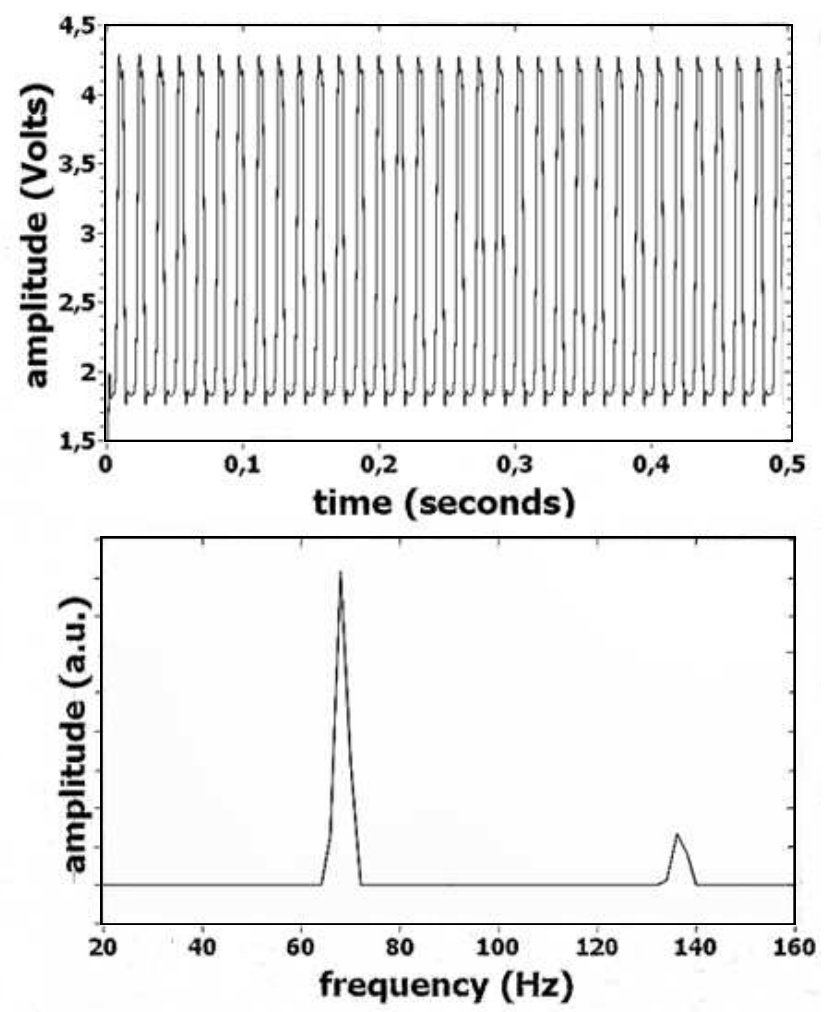

Figure 7: Upper picture: Reflected light intensity versus time in seconds (the data have an arbitrary offset). Lower picture: Fourier transformation of the reflected intensity.

The reflected intensity versus time as recorded by our data acquisition system is plotted in the upper plot of Fig. 7. Also shown, in the lower plot, is the corresponding Fourier transformation derived with the LabVIEW function 'Power Spectrum'. We use a peak finding algorithm (LabVIEW function 'Harmonic Distortion Analyzer') to determine the position of the fundamental mode, which is able to interpolate in steps 
of $2 \mathrm{~Hz}$ resulting from the sampling frequency of $8000 \mathrm{~Hz}$ and the sampling length of $0.5 \mathrm{~s}$. The measurement stops, when the peak finding algorithm has detected a frequency within a certain range. In our case, this range was chosen from $40 \mathrm{~Hz}$ to $90 \mathrm{~Hz}$ to find frequencies around $f^{\prime} \approx 2 \cdot 37 \mathrm{~Hz}$. The exact values to be chosen as upper and lower bounds depend on the variations incurred in the actual wire tensions.

Our data acquisition system samples the signal from the detector with $f_{0}=8000$ samples per second corresponding to a Nyquist frequency of $4000 \mathrm{~Hz}$. Typically, the oscillation of the reflected laser light is stationary after $1 \mathrm{~s}$ and dies out within less then ten seconds. Therefore, we have chosen to sample the signal for a few seconds and calculate the Fourier transform every 0.5 s i.e. every 4000 samples (Fig. 7 bottom).

In order to suppress high frequency noise, we filter the signal with a third order Butterworth low-pass with a cutoff frequency of $100 \mathrm{~Hz}$ in LabVIEW before the power spectrum of the signal is being computed. The cutoff frequency is also chosen such that harmonics above the signal frequency of the fundamental mode are suppressed.

The choice of this particular filter has no special reason, but it satisfies our requirements nicely. Using a software filter from LabVIEW's library instead of a hardware filter maintained our flexibility for different edge frequencies, since we commissioned the laser sensor while the first electrode modules for KATRIN were built and the design of the last modules was still undefined.

In order to determine the precision of the tension measurement, we varied the tension of a $176 \mathrm{~cm}$ long test wire with $0.3 \mathrm{~mm}$ diameter and measured the frequency with our laser sensor (Fig. 8). The tension was measured independently with a calibrated load cell (WRC-01 USB, model DBBP-20 by Weiss Robotics). Following Eq. 5, we fit the resulting data with a parabola

$$
F(f)=a \cdot f^{\prime 2},
$$

where $a$ is a free parameter. As mentioned above the measured frequency $f^{\prime}$ corresponds to twice the oscillation frequency of the wire $f$. Assuming a constant uncertainty of the force $\sigma_{F}$ the least-squares fit results in $\sigma_{F}=0.04 \mathrm{~N}$ for the uncertainty of the tension determination (Fig. 8). The measured deviations from the fit (Fig. 8 bottom) confirm the precision of the method.

The fit result $a=(1.587 \pm 0.0002) \cdot 10^{-3} \mathrm{~N} / \mathrm{Hz}^{2}$ can be compared to the expectation from Eq. (5).

One has to keep in mind that there are uncertainties of the parameters $l, A$ and $\rho$. The reflection of the standing wave at the ends of the wire is not necessarily ideal. We do not know the uncertainty of $\rho=7980 \mathrm{~kg} / \mathrm{m}^{3}$ but could measure $l$ and the wire diameter $d$ directly.

The measured parameters $(l=1.76 \pm 0.003 \mathrm{~m}$ and $d=0.29 \pm 0.005 \mathrm{~mm})$ imply a theoretical value $a=(1.633 \pm 0.057) \cdot 10^{-3} \mathrm{~N} / \mathrm{Hz}^{2}$. I.e. the theoretical prediction is unsignificantly larger than the observation. 

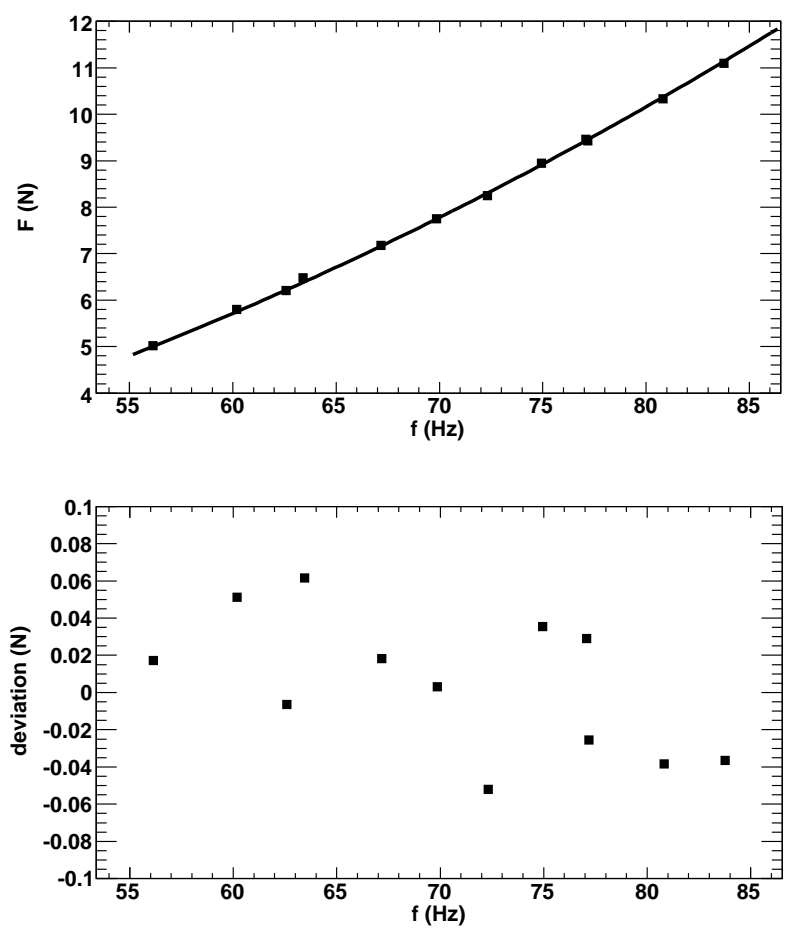

Figure 8: Parabolic fit to the measured wire tensions (top) assuming a constant uncertainty of $\Delta F=0.04 \mathrm{~N}$ and the corresponding deviations from the parabolic leastsquares fit (bottom). 


\section{Error Sources and Limitations}

For the stainless steel wires under scrutiny (diam. $0.2 \mathrm{~mm}-0.3 \mathrm{~mm}, l \approx 1.8 \mathrm{~m}$, $\rho=7980 \mathrm{Kg} / \mathrm{m}^{3}$ ), we observe that the tension can no longer be determined when it is less than $\approx 1 \mathrm{~N}$. Around that value, the intensity variation of the reflected laser light is no longer periodical.

An important specification for our sensor is a maximum weight of $250 \mathrm{~g}$. The weight of the sensor causes the arm of the CMM to sag by $30 \mu \mathrm{m}$ when fully extended $(1 \mathrm{~m})$. This sag is tolerable for us, and can be compensated for.

The precision of the position measurements depends on the correct alignment of the two laser beams. When the laser beams are not perfectly aligned with the measurement plane, the beams hit the wire a slightly too early or too late, when the sensor moves across a wire. This leads to an error in the determination of $x_{\text {green }}$ and $x_{\text {red }}$ leading to a systematic error of the wire position.

Suppose that the red laser is correctly aligned, but the green laser is displaced by $0.5 \mathrm{~mm}$ with respect to the measurement plane. Let the measured wire lie in the $(\mathrm{x}, \mathrm{z})-$ plane and both angles $\alpha$ and $\beta$ are $35^{\circ}$. Let us further assume, that the measured wire has an inclination of $5^{\circ}$ with respect to the z-axis. The resulting errors due to this misalignment will be:

$$
\begin{aligned}
& \left|\Delta x_{\text {wire }}\right|=\left|\frac{\partial x_{\text {wire }}}{\partial x_{\text {green }}} \Delta x_{\text {green }}\right|=0.02 \mathrm{~mm} \\
& \left|\Delta y_{\text {wire }}\right|=\left|\frac{\partial y_{\text {wire }}}{\partial x_{\text {green }}} \Delta x_{\text {green }}\right|=0.02 \mathrm{~mm}
\end{aligned}
$$

\section{Comparison with other Methods}

There are numerous methods which can be used to measure wire tensions and positions from a distance. In a well-known method for measuring the wire tension, an external magnetic field is applied and mechanical oscillations of the wire are induced by passing an alternating current through it. As long as no special techniques are employed one has to scan a frequency range in order to find the resonance frequency of the wire, which provides the tension measurement. This process can take several minutes [3]. This time can be drastically shortened using a sudden excitation of the wire in the magnetic field with a current pulse [4] or an electrostatic excitation through capacitive coupling [5]. In any case, one still needs to connect the wires to an external circuit, which is still time-consuming. Moreover, it could lead to a damage of the wires.

It has also been reported that one can excite wire oscillations with a small hammer, even if the wire thickness is only $30 \mu \mathrm{m}$, without causing any damage [6]. It could be difficult to adapt the corresponding hardware to changing geometries. Thus the real advantage of our excitation method is not that we avoid damage, but that we avoid the necessity to adapt mechanics to changing module geometries.

Other authors describe wire position measurements with a laser diode with an accuracy of better than $10 \mu \mathrm{m}$ for relative wire separations of thin wires $(<76 \mu \mathrm{m}$ diameter $)$ in a wire chamber but with a principle which is restricted to one dimension [7]. There is also an example [8] of a measurement with two CCDs in 2 dimensions with an accu- 
racy of $O(1) \mu \mathrm{m}$ but from a smaller distance of $\leq 40 \mathrm{~mm}$. The last method is the most precise method in two dimensions of which we are aware, however, it cannot be used to measure wire tensions.

\section{Conclusion}

We developed a new two-dimensional laser sensor for the measurement of parameters of thin wires. The key feature of our novel sensor is the combination of a twodimensional position measurement with a tension measurement at a distance of $150 \mathrm{~mm}$ with respect to the wires. Both the position and the tension measurements can be performed within a few seconds without touching the wire.

The laser sensor is moved by a precise three-dimensional coordinate table and the intensity of the reflected light is recorded. With this a position resolution of better than $10 \mu \mathrm{m}$ has been achieved. For the measurement of the wire tension, oscillations are excited by a puff of gas blown at the wire, and the intensity variation of the reflected light is recorded. The fundamental frequency of this variation is found by means of a fast Fourier transform and yields the wire tension with a precision of $0.04 \mathrm{~N}$. An important property for our application is the light-weight construction of the laser sensor $(<250 \mathrm{~g})$. We apply this wire measurement system for the quality assurance of the wire electrode system of the KATRIN neutrino mass experiment [1]. In a second version of the sensor we have now enlarged the detector lenses to a diameter of $40 \mathrm{~mm}$ in order to increase the angle of acceptance to $\pm 10^{\circ}$ to measure wires non-perpendicular to the laser plane.

In practice, our sensor needs $\approx 20$ minutes to measure the tensions of all 120 wires of a module and $\approx 30$ minutes to scan 120 wire position in one plane. A large fraction of the time, our CMM has to move the sensor from one wire to another. As soon as the CMM has positioned the sensor on a wire, it takes between two and four seconds to obtain a result for a wire tension. A position measurement, i.e., a scan across a wire, takes $\approx 10 \mathrm{~s}$. If it is required to speed up the measurement process, a faster CMM machine could be used, up to the limit of vibration damping of the sensor.

\section{Acknowledgment}

The authors would like to thank A. Gebel for his help as well as the mechanics and electronics workshops of our institute for their support. We would like to thank H. Gottschlag (ALICE Collaboration), who has provided us with a 1-dimensional sensor for prototype experiments. This sensor was developed for the measurement of wire tensions of TRD readout chambers of the ALICE experiment [13]. He also provided support for the first measurements, which evolved into the idea for our new sensor.

We also thank J.P. Wessels for carefully reading and correcting the article. This project is funded by the German Ministry of Research and Education (BMBF). 


\section{References}

[1] KATRIN Design Report, FZKA7090 (2004).

[2] US Federal Standard 209E, officially cancelled but still widely used, replaced by ISO 14644

[3] S. Bhadra, S. Errede, L. Fishback, H. Keutelian and P. Schlabach, Nucl. Instrum. Meth. A 269 (1988) 33.

[4] M. Calvetti, G. Piano Mortari, A. Placci and M. Rijssenbeek [UA1 Collaboration], Nucl. Instrum. Meth. 174 (1980) 285.

[5] P. Ciambrone, et. al. Nucl. Instrum. Meth. A 545 (2005) 156.

[6] W. Baldini et. al. A Laser Based Instrument for MWPC Wire Tension Measurement, Available:

http://cdsweb.cern.ch/record/1055333/files/lhcb-2007-120.pdf

[7] D.S. Carman, D. Bilodeau, L.C. Bland, A. Eads, T. Rinckel, K. Solberg Nuclear Instruments and Methods in Physics Research Section A, v. 394, p. 409-414.

[8] K. Hashemi, Open Source Instruments, Inc. 130 Mount Auburn Street Watertown, MA 02472 Wire Position Sensor.

Available: http://www.opensourceinstruments.com/

[9] Maxon Motor GmbH, Germany, http://www.maxonmotor.de

[10] Filters by Baader Planetarium, Germany (item number 2458304 and 2458307)

[11] Card number NI PCI-6014 by National Instruments

[12] LabVIEW Version 8.0, National Instruments, Inc. Austin TX, http://www.ni.com

[13] GSI Scientific Report 2003, p.64

Available:

http://www.gsi.de/informationen/wti/library/scientificreports.html 